\title{
Non-modal stability of round viscous jets
}

\author{
S. A. Boronin ${ }^{1} \uparrow$, J. J. Healey ${ }^{2}$ and S. S. Sazhin ${ }^{1}$ \\ ${ }^{1}$ Sir Harry Ricardo Laboratories, School of Computing, Engineering and Mathematics, \\ University of Brighton, Brighton BN2 4GJ, UK \\ ${ }^{2}$ Department of Mathematics, Keele University, Keele, Staffs ST5 5BG, UK
}

(Received 19 March 2012; revised 31 July 2012; accepted 9 October 2012)

\begin{abstract}
Hydrodynamic stability of round viscous fluid jets is considered within the framework of the non-modal approach. Both the jet fluid and surrounding gas are assumed to be incompressible and Newtonian; the effect of surface capillary pressure is taken into account. The linearized Navier-Stokes equations coupled with boundary conditions at the jet axis, interface and infinity are reduced to a system of four ordinary differential equations for the amplitudes of disturbances in the form of spatial normal modes. The eigenvalue problem is solved by using the orthonormalization method with Newton iterations and the system of least stable normal modes is found. Linear combinations of modes (optimal disturbances) leading to the maximum kinetic energy at a specified set of governing parameters are found. Parametric study of optimal disturbances is carried out for both an air jet and a liquid jet in air. For the velocity profiles under consideration, it is found that the non-modal instability mechanism is significant for non-axisymmetric disturbances. The maximum energy of the optimal disturbances to the jets at the Reynolds number of 1000 is found to be two orders of magnitude larger than that of the single mode. The largest growth is gained by the streamwise velocity component.
\end{abstract}

Key words: jets, gas/liquid flow, instability

\section{Introduction}

The problem of jet instability and break-up was first studied within the framework of the classical linear hydrodynamic stability theory by Rayleigh (1945). Since then, the practical importance of the problem has given rise to a large number of theoretical and experimental investigations (Lin \& Reitz 1998; Söderberg \& Alfredsson 1998; Sazhin et al. 2008; Turner et al. 2012b).

To the best of the authors' knowledge, stability of round jets has been analysed only within the framework of the modal approach, which is based on the analysis of small disturbances to the main flow in the form of a single normal mode. There are several examples of shear flows, where the classical stability theory fails to predict flow transition even qualitatively. For example, it is experimentally observed that the flow in a round pipe loses stability at sufficiently large Reynolds numbers (Reynolds 1883), which contradicts the results of the classical hydrodynamic stability theory (Drazin

$\dagger$ Present address: Institute of Mechanics, Lomonosov Moscow State University, Moscow, 119192 Michurinskii pr. 1, Russian Federation. Email address for correspondence: s.sazhin@brighton.ac.uk 
$\&$ Reid 1983). The instability scenario does not depend on the level of turbulence in the main flow while it is sufficiently small (Klingmann 1992). The discrepancy between the experiments and theoretical stability studies gave rise to so-called 'bypass' approaches, which describe the transition of shear flows without taking into account the modal (exponential) structure of disturbances. It is assumed that there are some initial disturbances to the main flow that can grow and reach the amplitude required to trigger nonlinear instability or provide basic states for secondary instabilities even if all normal modes decay.

About 40 years ago, it was shown theoretically that there are disturbances to all plane-parallel shear flows, which grow linearly during the limited interval of time ('liftup' or 'vortex-tilting' mechanism) (Ellingsen 1975; Landahl 1980). The non-modal (algebraic) growth of small disturbances can be described based on the analysis of linear differential operators, which govern the evolution of small disturbances (e.g. coupled Orr-Sommerfeld and Squire operators for normal velocity and normal vorticity (Butler \& Farrel 1992)). These operators are non-Hermitian, which means that their system of eigenvectors (normal modes or basic solutions) are not orthogonal. It is not sufficient to consider only the eigenvector with the largest growth rate in order to describe the evolution of all small disturbances over the limited time interval, the whole spectrum should be used. Even if all eigenvectors decay (the flow is stable in the classical sense), there still can be some combinations of eigenvectors which grow significantly during a limited interval of time before they eventually decay. This non-modal or transient growth can trigger the flow transition (Schmid \& Henningson 2001). The combinations of normal modes with the largest energy at a certain time instant (for the temporal approach) or location downstream (for the spatial approach) are called 'optimal disturbances'.

The non-modal or transient growth of disturbances has been studied for a number of typical shear flows (Butler \& Farrel 1992; Reshotko \& Tumin 2001; Tumin \& Reshotko 2001). It has been found that the optimal disturbances to boundary layer, plane Poiseuille, Couette and pipe flows are streamwise-elongated structures similar to those found in experiments. To the best of the authors' knowledge, the idea of algebraic instability has not been applied to the problem of round jet break-up, while streamwise ligaments and other streamwise patterns are observed in experiments on jet flows (Hoyt \& Taylor 1977; Lasheras \& Hopfinger 2000; Marmottant \& Villermaux 2004). De Luca (2001) has studied optimal disturbances to a plane viscous jet and has not found any significant non-modal growth. The optimal-to-single mode energy ratio has not exceeded 2.5. The algebraic instability of a wall jet was investigated by Levin et al. (2005). It has been found that the energy of stationary optimal disturbances (with zero frequency) is three orders of magnitude larger than that of the first mode with the same initial energy. The non-modal growth of disturbances to a sheared interface has been analysed by Yecko \& Zaleski (2005). The significant temporal growth of streamwise-independent disturbances has been found. The maximum kinetic energy of optimal disturbances is several orders of magnitude larger than its initial value, and non-modal growth increases with an increase in the Reynolds number. The shape of optimal disturbance has been studied and it has been found that there is a significant amplification of streamwise velocity indicating the origination of streamwise 'streaky' structures.

The present study is aimed at the analysis of the algebraic instability of two types of jets, air jets in air and liquid jets in air, which is expected to improve our understanding of the problem of jet break-up. The investigation of the latter problem is important for various environmental and engineering applications, including diesel 
engines (Sazhin et al. 2003; Crua et al. 2004; Sazhin et al. 2008; Turner et al. 2012b). The understanding of the jet break-up would help us to control it, although the latter problem is beyond the scope of this paper. Several types of main flow velocity profiles are considered, which correspond to different distances from the orifice and different nozzle lengths. In order to perform a thorough non-modal analysis, the linear stability problem is considered in the most general way: the disturbances are assumed to be three-dimensional and non-axisymmetric. In contrast to the algebraic instability studies mentioned above, we consider spatially growing disturbances instead of temporal ones. It allows us to study the spatial evolution of disturbances in a downstream direction and evaluate the possible effect of non-modal growth on break-up length of jets.

\section{Problem formulation}

\subsection{Governing equations}

We consider an isothermal flow of fluid, injected from a round orifice, into another fluid. Two types of jets are studied: a fluid jet released into the identical fluid (we will refer to it as a 'submerged jet') and a fluid jet surrounded by a fluid with different physical properties ('liquid jet in air'). Both fluids are incompressible and Newtonian. The system of non-dimensional governing equations in the cylindrical coordinate system $(z, r, \theta)$ with the $z$-axis directed along the flow is presented as (Batchelor 1967)

$$
\begin{gathered}
\frac{\partial u_{\alpha}}{\partial z}+\frac{1}{r} \frac{\partial v_{\alpha} r}{\partial r}+\frac{1}{r} \frac{\partial w_{\alpha}}{\partial \theta}=0 \\
\frac{\partial u_{\alpha}}{\partial t}+u_{\alpha} \frac{\partial u_{\alpha}}{\partial z}+v_{\alpha} \frac{\partial u_{\alpha}}{\partial r}+\frac{w_{\alpha}}{r} \frac{\partial u_{\alpha}}{\partial \theta}=-\frac{\partial p_{\alpha}}{\partial z}+\frac{1}{R e_{\alpha}} \Delta u_{\alpha} \\
\frac{\partial v_{\alpha}}{\partial t}+u_{\alpha} \frac{\partial v_{\alpha}}{\partial z}+v_{\alpha} \frac{\partial v_{\alpha}}{\partial r}+\frac{w_{\alpha}}{r} \frac{\partial v_{\alpha}}{\partial \theta}-\frac{w_{\alpha}^{2}}{r}=-\frac{\partial p_{\alpha}}{\partial r}+\frac{1}{R e_{\alpha}}\left(\Delta v_{\alpha}-\frac{2}{r^{2}} \frac{\partial w_{\alpha}}{\partial \theta}-\frac{v_{\alpha}}{r^{2}}\right) \\
\frac{\partial w_{\alpha}}{\partial t}+u_{\alpha} \frac{\partial w_{\alpha}}{\partial z}+v_{\alpha} \frac{\partial w_{\alpha}}{\partial r}+\frac{w_{\alpha}}{r} \frac{\partial w_{\alpha}}{\partial \theta}+\frac{v_{\alpha} w_{\alpha}}{r} \\
=-\frac{1}{r} \frac{\partial p_{\alpha}}{\partial \theta}+\frac{1}{R e_{\alpha}}\left(\Delta w_{\alpha}+\frac{2}{r^{2}} \frac{\partial v_{\alpha}}{\partial \theta}-\frac{w_{\alpha}}{r^{2}}\right)
\end{gathered}
$$

where

$$
\left.\begin{array}{l}
\Delta=\frac{\partial^{2}}{\partial z^{2}}+\frac{1}{r} \frac{\partial}{\partial r}\left(r \frac{\partial}{\partial r}\right)+\frac{1}{r^{2}} \frac{\partial^{2}}{\partial \theta^{2}}, \quad R e_{l}=\frac{U L \rho_{l}}{\mu_{l}}, \quad R e_{g}=R e_{l} \frac{\eta}{\zeta}, \\
\eta=\frac{\rho_{g}}{\rho_{l}}, \quad \zeta=\frac{\mu_{g}}{\mu_{l}},
\end{array}\right\}
$$

where the subscript $\alpha=l, g$ indicates the jet fluid (liquid) and surrounding fluid (gas), respectively, $U$ is the maximum velocity of the jet, $L$ is the length scale (radius of the orifice), $\rho_{\alpha}$ and $\mu_{\alpha}$ are densities and viscosities of the fluids, pressures $p$ are scaled by the corresponding dynamic pressures $\rho_{\alpha} U^{2}$ (different in jet liquid and surrounding gas). We have chosen the following independent governing parameters: $R e_{l}$ is the Reynolds number based on jet liquid parameters; $\eta$ and $\zeta$ are gas-to-liquid density and viscosity ratios.

System (2.1)-(2.4) is coupled with the following boundary conditions:

(i) kinematic conditions at the axis $r=0$ (all flow parameters are finite):

$$
\left|\boldsymbol{v}_{l}\right|<\infty, \quad p_{l}<\infty, \quad \forall z, t \geqslant 0 ;
$$


(ii) kinematic conditions at the interface $r=H(z, \theta, t)$ (fluid particles at the interface do not leave it)

$$
\frac{\partial H}{\partial t}+u_{\alpha} \frac{\partial H}{\partial z}+v_{\alpha} \frac{\partial H}{\partial r}+\frac{w_{\alpha}}{r} \frac{\partial H}{\partial \theta}=0, \quad \alpha=l, g ;
$$

(iii) continuity of velocity and jump in normal stress due to capillary force acting at the interface $r=H(z, \theta, t)$

$$
\boldsymbol{v}_{l}=\boldsymbol{v}_{g}, \quad \boldsymbol{s}_{l}-\eta \boldsymbol{s}_{g}=\boldsymbol{R}
$$

where

$$
\begin{gathered}
\boldsymbol{s}_{\alpha}=-p_{\alpha} \boldsymbol{n}+\frac{1}{R e_{\alpha}} \sum_{i, j=1}^{3}\left(\nabla_{i} v_{\alpha, j}+\nabla_{j} v_{\alpha, i}\right) n_{i} \boldsymbol{e}_{j}, \quad \boldsymbol{R}=-\frac{1}{W e} \boldsymbol{n} \operatorname{div} \boldsymbol{n}, \\
W e=\frac{\rho_{l} U^{2} L}{\sigma}, \quad \boldsymbol{n}=\frac{\nabla H}{|\nabla H|},
\end{gathered}
$$

$\boldsymbol{s}_{l}, \boldsymbol{s}_{g}$ are jet liquid and ambient gas stresses at the interface; $\boldsymbol{R}$ is the capillary force; $\boldsymbol{n}=\left\{n_{1}, n_{2}, n_{3}\right\}$ is the unit vector normal to the interface; $v_{\alpha, i}$ are velocity components of the fluids; $\sigma$ is the surface tension; $\boldsymbol{e}_{i}(i=1,2,3)$ are basis vectors; $W e$ is the Weber number; stresses $s_{\alpha}$ are scaled by the corresponding dynamic pressures $\rho_{\alpha} U^{2}$ similar to (2.2)-(2.4);

(iv) conditions of decay at the infinity $r \rightarrow \infty$

$$
\boldsymbol{v}_{g} \rightarrow 0, \quad p_{g}<\infty
$$

\subsection{The base flow velocity profiles}

The velocity distribution in a stationary round axisymmetric liquid jet in air depends on the velocity profile at the orifice, which in turn is determined by the length of the nozzle $L_{n}$ (Duda \& Vrentas 1967; Söderberg \& Alfredsson 1998). If $L_{n}$ is small compared with a typical relaxation length of the velocity profile in the pipe $L_{c} \sim a R e$, where $a$ is the pipe radius, then the velocity profile at the orifice is nearly uniform. In the opposite case of a sufficiently long nozzle, the velocity distribution at the orifice is close to the Hagen-Poiseuille parabolic profile. By varying the nozzle length, it is possible to obtain different velocity profiles at the orifice with the shapes somewhere between these two limiting cases.

The process of velocity relaxation in round jets has been studied by a number of authors including Duda \& Vrentas (1967) and Shkadov \& Sisoev (1996). A non-uniform velocity profile of the jet fluid at the orifice tends to become uniform downstream from the orifice, as the velocity at the interface is subject to a momentum exchange with the core of the jet. It was found that for horizontal jets at sufficiently high Reynolds numbers $(R e>200)$, the dynamics of velocity distribution across the jet is affected only by the surface tension determined by the Weber number. The relaxation length of the jet radius is significantly shorter than that of the jet velocity, which is of the order of $a R e$. It was shown that the relaxation process is quite intense in the vicinity of the orifice; both the jet radius and the velocity at the interface vary significantly over the near-orifice region with the length of the order of 0.01aRe. The relaxation process is slow at $0.01 R e<z<0.1 R e$ (the coordinate $z$ is scaled by the radius of the jet, introduced in the above set of governing equations). This allows us to consider the local stability of the jet, avoiding the dependence of the velocity distribution in the main flow on the downstream location $z$. Note that the focus of 


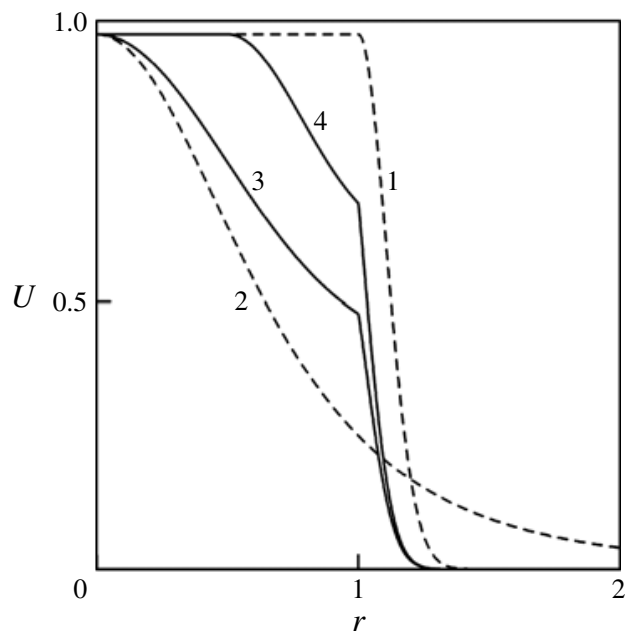

FIGURE 1. Jet velocity profiles: curves 1 and 2 show profiles $U_{1,2}$ for the submerged jet $\left(\delta=0.5\right.$ ); curves 3 and 4 show profiles $U_{3,4}$ for the liquid jet in air, $\zeta=0.1$ (parameters of the profiles are presented in appendix A).

the current study is on the analysis of the possibility for non-modal growth of small disturbances to round jets, while the effect of the velocity profile variation in the downstream direction on transient growth is beyond the scope of our paper.

We consider the stability of a cylindrical jet with velocity $V_{\alpha}$ and pressure $P_{\alpha}$ in the following form:

$$
V_{\alpha}=\left\{U_{\alpha}(r), 0,0\right\}, \quad P_{\alpha}=\text { const. }
$$

Functions (2.12) satisfy system (2.1)-(2.4) subject to boundary conditions (2.6)-(2.11).

In the case of a submerged jet, the velocity and the pressure are subject to conditions (2.6) and (2.11) only. We consider two velocity profiles $U_{1,2}$, namely the 'top-hat' velocity profile, which mimics the velocity distribution in the submerged jet close to the orifice, and the 'smooth' profile (Landau \& Lifshitz 1959), which is the asymptotic distribution of the velocity in the far-downstream region of the submerged jet (figure 1, curves 1 and 2):

$$
\begin{gathered}
U_{1}(r)=\left\{\begin{array}{ll}
1, & r \leqslant 1 \\
\exp \left\{-(r-1)^{2} / \delta^{2}\right\}, & r>1
\end{array},\right. \\
U_{2}(r)=\frac{1}{\left(1+r^{2}\right)^{2}},
\end{gathered}
$$

where parameter $\delta$ determines the steepness of the velocity profile. In our study we consider $\delta=0.5$.

For the liquid jet in air, boundary conditions (2.6)-(2.11) can be written as

$$
U_{g}(r) \rightarrow 0, r \rightarrow \infty ; \quad U_{l}(1)=U_{g}(1), U_{l}^{\prime}(1)=\zeta U_{g}^{\prime}(1) ; \quad\left|U_{l}(r)\right|<\infty, r \rightarrow 0 .
$$

We consider two velocity profiles $U_{3,4}$ given in appendix A, which satisfy conditions (2.15) and determine the velocity distributions in the cross-section of the liquid jet in air, at a certain distance from the orifice. These are shown in figure 1, curves 3 and 4. 
Profile $U_{3}$ refers to the case when the nozzle is long enough to develop the Hagen-Poiseuille flow at the orifice, while $U_{4}$ refers to a shorter nozzle. As mentioned earlier, there is a rapid relaxation of the jet velocity in the vicinity of the orifice. We have chosen intermediate velocity profiles, typical for the region away from the nozzle.

In various engineering applications, jets are unsteady (e.g. in internal combustion engines the fuel jet is accelerated during a limited interval of time before the volumetric flux of the fuel levels off). Linear stability analysis of unsteady flows is significantly more complex compared with that of stationary flows (Schmid \& Henningson 2001; Turner et al. 2012a). The time scale of the disturbance growth and propagation should be small compared with the time scale of acceleration/deceleration of the main flow. Otherwise, the normal modes, predicted by the classical local stability approach, fail to describe the evolution of small perturbations to the nonstationary jet.

\subsection{Formulation of the linear stability problem}

We consider the stability of axisymmetric cylindrical jets with profiles $U_{1-4}$. Note that the origin of the coordinate system $z=0$ is placed at a certain distance downstream from the nozzle, where the cross-flow distribution of the velocity corresponds to specified profiles. The base flow described by (2.12) is subject to small three-dimensional disturbances $\boldsymbol{v}_{\alpha}=\left\{u_{\alpha}, v_{\alpha}, w_{\alpha}\right\}$, and $p_{\alpha}$. The linearized system of governing equations is written as

$$
\begin{gathered}
\frac{\partial u_{\alpha}}{\partial z}+\frac{1}{r} \frac{\partial v_{\alpha} r}{\partial r}+\frac{1}{r} \frac{\partial w_{\alpha}}{\partial \theta}=0 \\
\frac{\partial u_{\alpha}}{\partial t}+U_{\alpha} \frac{\partial u_{\alpha}}{\partial z}+v_{\alpha} U_{\alpha}^{\prime}=-\frac{\partial p_{\alpha}}{\partial z}+\frac{1}{R e_{\alpha}} \Delta u_{\alpha}, \\
\frac{\partial v_{\alpha}}{\partial t}+U_{\alpha} \frac{\partial v_{\alpha}}{\partial z}=-\frac{\partial p_{\alpha}}{\partial r}+\frac{1}{R e_{\alpha}}\left(\Delta v_{\alpha}-\frac{2}{r^{2}} \frac{\partial w_{\alpha}}{\partial \theta}-\frac{v_{\alpha}}{r^{2}}\right), \\
\frac{\partial w_{\alpha}}{\partial t}+U_{\alpha} \frac{\partial w_{\alpha}}{\partial z}=-\frac{1}{r} \frac{\partial p_{\alpha}}{\partial \theta}+\frac{1}{R e_{\alpha}}\left(\Delta w_{\alpha}+\frac{2}{r^{2}} \frac{\partial v_{\alpha}}{\partial \theta}-\frac{w_{\alpha}}{r^{2}}\right) .
\end{gathered}
$$

Linearized boundary conditions at the axis (2.6) and at infinity (2.11) remain the same, while linearized conditions at the disturbed interface $r=1+h(z, \theta, t)(2.7)$, (2.8) are written at the undisturbed surface $r=1$ (using the Taylor series) as

$$
\begin{gathered}
\frac{\partial h}{\partial t}+U_{\alpha} \frac{\partial h}{\partial z}=v_{\alpha}, \quad \alpha=l, g \\
u_{l}+h U_{l}^{\prime}=u_{g}+h U_{g}^{\prime}, \quad v_{l}=v_{g}, \quad w_{l}=w_{g} \\
\frac{\partial v_{l}}{\partial z}+\frac{\partial u_{l}}{\partial r}+h U_{l}^{\prime \prime}=\zeta\left(\frac{\partial v_{g}}{\partial z}+\frac{\partial u_{g}}{\partial r}+h U_{g}^{\prime \prime}\right) \\
-p_{l}+\frac{2}{R e_{l}} \frac{\partial v_{l}}{\partial r}=-\eta p_{g}+\frac{2 \zeta}{R e_{l}} \frac{\partial v_{g}}{\partial r}+\frac{1}{W e}\left(h+\frac{\partial^{2} h}{\partial z^{2}}+\frac{\partial^{2} h}{\partial \theta^{2}}\right) \\
\frac{\partial w_{l}}{\partial r}+\frac{\partial v_{l}}{\partial \theta}-w_{l}=\zeta\left(\frac{\partial w_{g}}{\partial r}+\frac{\partial v_{g}}{\partial \theta}-w_{g}\right) .
\end{gathered}
$$

We are interested in the solutions to the linearized system of governing equations in the form of travelling waves with a real frequency $\omega$, integer azimuthal number $m$ and 
complex wavenumber $k$ :

$$
\boldsymbol{Q}(z, r, \theta, t)=\boldsymbol{q}(r) \exp \{\mathrm{i}(k z+m \theta-\omega t)\},
$$

where vector $\boldsymbol{Q}$ stands for $\left(u_{\alpha}, v_{\alpha}, w_{\alpha}, p_{\alpha}\right)$.

If we substitute expressions (2.25) into (2.16)-(2.24), we obtain the system of four ordinary differential equations coupled with homogeneous boundary conditions. Following the standard approach to the Cauchy problem and using the continuity (2.16) to eliminate $v^{\prime \prime}(r)$, the system can be rewritten in the form of six first-order differential equations:

$$
\boldsymbol{L q}_{\alpha}=0, \quad \boldsymbol{A} \boldsymbol{q}_{l}(0)=0, \quad \boldsymbol{B} \boldsymbol{q}_{l}(1)=\boldsymbol{C q}_{g}(1), \quad \boldsymbol{D} \boldsymbol{q}_{g}(\infty)=0, \quad \boldsymbol{q}=\left\{u, v, w, p, u^{\prime}, w^{\prime}\right\}
$$

where $\boldsymbol{L}$ is a $6 \times 6$ first-order linear differential operator, $\boldsymbol{A}, \boldsymbol{B}, \boldsymbol{C}$ and $\boldsymbol{D}$ are $6 \times 6$ matrices of boundary conditions. The explicit form of (2.26) is presented in appendix B. Note that all parameters of liquid at interface $\boldsymbol{q}_{l}(1)$ can be expressed in terms of gas parameters $\boldsymbol{q}_{g}(1)$ and vice versa. Therefore, (2.26) determine a two-point boundary-value problem rather than a three-point boundary-value problem.

Eigenvalue problem (2.26) leads to a set of eigenfunctions and eigenvalues for a given set of governing parameters $\operatorname{Re}_{l}, \zeta, \eta, W e, m$ and $\omega$ :

$$
\left\{\boldsymbol{q}_{l, i}, \boldsymbol{q}_{g, i}, k_{i}\right\}, \quad i=1 \ldots \infty .
$$

Since the jet flow is unbounded in the $r$-direction, there is also a continuum set of modes determined by eigenvalue problem (2.26), and it has to be considered while solving the initial-value problems for the linearized system of governing equations (2.16)-(2.19) (Schmid \& Henningson 2001). It is a common approach to represent the continuous spectrum by discrete eigenvalues and consider only a finite number $N$ of modes with the highest increments of growth (Butler \& Farrel 1992). This can be done by replacing the infinite flow domain $r>0$ with the finite flow domain $0<r<R$, where $R$ is sufficiently large. The number of modes $N$ and the size of the computational domain $R$ are determined by the required accuracy of calculations and cannot be a priori specified.

\subsection{Non-modal stability and optimal disturbances}

We assume that every $t$ - and $\theta$-periodic solution to system (2.16)-(2.19) with a given frequency $\omega$ and azimuthal mode number $m$ can be approximated by a linear combination of $N$ normal modes (2.25) (Schmid \& Henningson 2001):

$$
\boldsymbol{q}_{\alpha}(r, z, \theta, t)=\sum_{j=1}^{N}\left\{\gamma_{j} \boldsymbol{q}_{\alpha, j}(r) \exp \left(\mathrm{i} k_{j} z\right)\right\} \exp \{\mathrm{i}(m \theta-\omega t)\},
$$

where complex vector $\boldsymbol{\gamma}$ is the spectral projection of the disturbance into the set of normal modes.

In order to evaluate the growth of disturbances (2.28), the appropriate norm $E(\boldsymbol{q})$ should be introduced. The usual choice for the norm in studies of transient growth in single-fluid flows is the kinetic energy of the disturbance confined to single periods in $\theta$ and $t$ (Butler \& Farrel 1992; Reshotko \& Tumin 2001; Tumin \& Reshotko 2001; Yecko \& Zaleski 2005). When studying the transient growth in two-fluid flows it is necessary to include an additional term related to the interfacial energy into the expression for the norm, otherwise the calculations converge very poorly 
(Renardy 1987; van Noorden et al. 1998; South \& Hooper 1999; Malik \& Hooper 2007). We consider the following expression for the energy norm:

$$
E=F+\eta G+I,
$$

where

$$
\begin{gathered}
F=\frac{1}{2 a b} \int_{0}^{1} \int_{0}^{a} \int_{0}^{b}\left(\left\{\operatorname{Real}\left[u_{l}\right]\right\}^{2}+\left\{\operatorname{Real}\left[v_{l}\right]\right\}^{2}+\left\{\operatorname{Real}\left[w_{l}\right]\right\}^{2}\right) r \mathrm{~d} r \mathrm{~d} t \mathrm{~d} \theta \\
G=\frac{1}{2 a b} \int_{1}^{R} \int_{0}^{a} \int_{0}^{b}\left(\left\{\operatorname{Real}\left[u_{g}\right]\right\}^{2}+\left\{\operatorname{Real}\left[v_{g}\right]\right\}^{2}+\left\{\operatorname{Real}\left[w_{g}\right]\right\}^{2}\right) r \mathrm{~d} r \mathrm{~d} t \mathrm{~d} \theta \\
a=\frac{2 \pi}{\omega}, b=\frac{2 \pi}{m}, \\
I=\frac{1}{2 a b} \int_{0}^{a} \int_{0}^{b} M_{0}(\operatorname{Real}[h])^{2} \mathrm{~d} t \mathrm{~d} \theta
\end{gathered}
$$

Real[·] corresponds to the real part of variables, $R \gg 1$ is the outer boundary of the flow domain in the $r$-direction and $M_{0}>0$ is a real constant which determines the input of the interfacial energy term into the norm.

All components of linear combination (2.28) are $t$ - and $\theta$-periodic functions $Q$ with the following properties:

$$
\begin{gathered}
Q(z, r, \theta, t)=\tilde{q}(r, z) \exp (\mathrm{i}\{m \theta-\omega t\}), \quad \tilde{q}(r, z)=\sum_{j=1}^{N} \gamma_{j} q_{j}(r) \exp \left(\mathrm{i} k_{j} z\right), \\
(\operatorname{Real}\{Q\})^{2}=\left(\frac{Q+Q^{*}}{2}\right)^{2}=\frac{1}{4}\left(Q^{2}+\left\{Q^{*}\right\}^{2}\right)+\frac{1}{2} Q Q^{*} \\
Q Q^{*}=\tilde{q} \tilde{q}^{*}=\sum_{j, s=1}^{N} \gamma_{j}^{*} q_{j}^{*} q_{s} \gamma_{s} \exp \left(-\mathrm{i}\left\{\operatorname{Im}\left[k_{j}\right]+\operatorname{Im}\left[k_{s}\right]\right\}\right)
\end{gathered}
$$

where the asterisk denotes the complex conjugation.

Since $Q^{2}$ and $\left(Q^{*}\right)^{2}$ are $t$ - and $\theta$-periodic functions, their integrals involved in expressions (2.29) are equal to zero. Hence, using expressions (2.33)-(2.35), energy functional (2.29) can be presented as a positively defined quadratic form with respect to components of the spectral projection $\boldsymbol{\gamma}$ :

$$
E(z, \boldsymbol{\gamma})=\sum_{j, s=1}^{N} \gamma_{j}^{*} E_{j s} \gamma_{s}=\boldsymbol{\gamma}^{*} \boldsymbol{E}(z) \boldsymbol{\gamma}
$$

where

$$
\begin{gathered}
\boldsymbol{E}(z)=\boldsymbol{F}(z)+\eta \boldsymbol{G}(z)+M \boldsymbol{I}(z), \\
F_{j s}=\frac{1}{4} \int_{0}^{1}\left(u_{l, j}^{*} u_{l, s}+v_{l, j}^{*} v_{l, s}+w_{l, j}^{*} w_{l, s}\right) r \mathrm{~d} r \exp \left(-\mathrm{i}\left\{\operatorname{Im}\left[k_{j}\right]+\operatorname{Im}\left[k_{s}\right]\right\} z\right), \\
G_{j s}=\frac{1}{4} \int_{1}^{R}\left(u_{g, j}^{*} u_{g, s}+v_{g, j}^{*} v_{g, s}+w_{g, j}^{*} w_{g, s}\right) r \mathrm{~d} r \exp \left(-\mathrm{i}\left\{\operatorname{Im}\left[k_{j}\right]+\operatorname{Im}\left[k_{s}\right]\right\} z\right), \\
I_{j s}=h_{j}^{*} h_{s} \exp \left(-\mathrm{i}\left\{\operatorname{Im}\left[k_{j}\right]+\operatorname{Im}\left[k_{s}\right]\right\} z\right),
\end{gathered}
$$

$F_{j s}, G_{j s}$ and $I_{j s}$ are components of energy matrices $\boldsymbol{F}(z), \boldsymbol{G}(z)$ and $\boldsymbol{I}(z)$ evaluated at a downstream location $z$ (they are Gram matrices of eigenvectors $\boldsymbol{v}_{l, j}$, and $\boldsymbol{v}_{g, s}$, $j, s=1 \ldots N)$, parameter $M>0$ determines the magnitude of the interfacial energy 


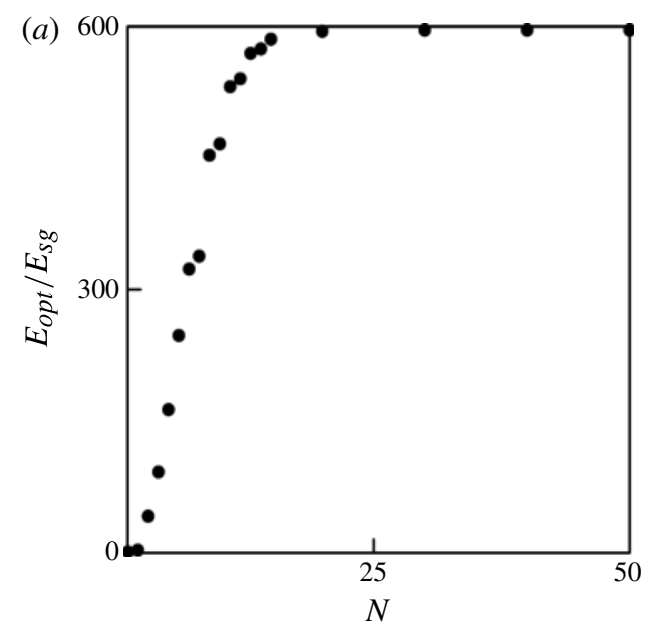

(b)

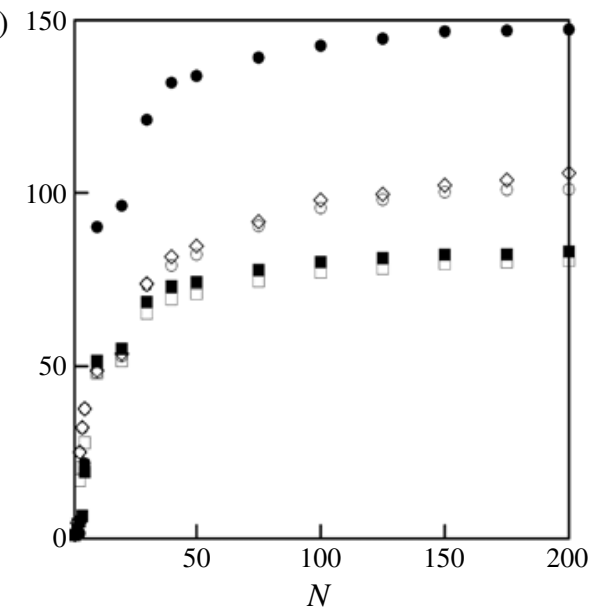

FIGURE 2. Convergence of transient growth calculations, maximum optimal-to-single mode energy ratio $E_{\text {opt }} / E_{s g}$ is plotted against the number of modes $N$ for submerged jet with 'smooth' velocity profile $U_{2}(a)$ and for liquid jet in air with velocity profile $U_{3}(b)$ at $\zeta=0.1$, $\eta=10^{-3}, W e=10^{3}$ (॰, $M=0 ; \mathbf{\square}, M=0.01 ; \square, M=0.1 ; \bullet, M=1 ; \diamond, M=0$ and $W e=\infty$ ). Here $\operatorname{Re}=10^{3}, m=1$ and $\omega=0.1$.

term. The effects of different choices of $M$ are explored in figure $2(b)$. Note that Renardy (1987), van Noorden et al. (1998), South \& Hooper (1999), Yecko \& Zaleski (2005) and Malik \& Hooper (2007) used a similar interfacial energy term proportional to the squared real part of the interface perturbation, the difference is in the choice of $M$.

The focus of our analysis is on finding a combination of eigenfunctions (2.28), which provides the maximum of the energy functional (2.36) at a fixed position $z$, given the energy of the disturbance at $z=0$ is unity:

$$
\max _{\gamma} E(z, \gamma)-?: E(0, \gamma)=1
$$

The solution $\gamma$ to the maximization problem (2.41) determines the disturbance with the highest possible energy at a given position $z$. For large $z$, the value of energy $E(z, \gamma)$ is determined by the least stable mode only, while at intermediate values of $z$, the influence of other modes can be significant and the algebraic instability of the flow can be initiated.

\section{Numerical solution}

\subsection{Algorithm of the numerical solution}

Eigenvalue problem (2.26) is solved numerically using the orthonormalization method proposed by Godunov (1961). Below we present the main ideas of the numerical algorithm for finding a system of normal modes and optimal disturbances.

The number of independent solutions to problem (2.26), which satisfy one of the boundary conditions either at $r \ll 1$ or $r \gg 1$, depends on the value of the azimuthal wavenumber $m$ (Morris 1976). There are one or two independent solutions for $m=0$ (torsional and meridional modes respectively), and three solutions for $m>0$. The asymptotics of these solutions at the specified regions can be found analytically (Garg \& Rouleau 1971; Morris 1976). In what follows, we present the orthonormalization 
algorithm for finding eigenvalues for problem (2.26) and consider the case of nonaxisymmetric disturbances $m=1$.

According to the theory of linear ordinary differential equations, the general solution to the boundary-value problem under consideration is presented as a linear combination of three fundamental solutions $\boldsymbol{q}_{j}, j=1,2,3$, which satisfy the boundary condition at the outer boundary $r \gg 1$ :

$$
\boldsymbol{q}(r)=a_{1} \boldsymbol{q}_{1}(r)+a_{2} \boldsymbol{q}_{2}(r)+a_{3} \boldsymbol{q}_{3}(r) .
$$

Three independent fundamental solutions $\boldsymbol{q}_{i}(r), i=1,2,3$, are obtained by the integration of (2.26) starting from the outer boundary of the flow $r=R$ towards the axis using the fourth-order Runge-Kutta method. The initial conditions $\boldsymbol{q}_{i}(R)$ are specified according to the asymptotical solution to (2.26) for $r \gg 1$, which involves Hankel functions of first and second orders depending on the governing parameters (Morris 1976). At the jet axis $r=\varepsilon, \varepsilon \ll 1$ the linear combination of solutions $\boldsymbol{q}_{i}(r)$, $i=1,2,3$, should be matched with a linear combination of asymptotical independent solutions to (2.26) at $r \ll 1$ (Garg \& Rouleau 1971). If the Reynolds number $R e$ is large, initially independent vectors $\boldsymbol{q}_{j}, j=1,2,3$, tend to become linear-dependent in the process of integration, which reduces the accuracy of calculations significantly. The orthonormalization of these vector functions is required in order to maintain reasonable accuracy (Godunov 1961). Vector functions $\boldsymbol{q}_{j}, j=1,2,3$, are orthonormalized using the standard Gram-Schmidt procedure, and the transformation matrices are stored in order to calculate the eigenfunctions.

Let the linear-independent asymptotic solutions to system (2.26), obtained analytically at $r \ll 1$, be $\boldsymbol{q}_{j}^{0}, j=1,2,3$, (Garg \& Rouleau 1971). Then, the boundary conditions at the axis can be presented as

$$
a_{1} \boldsymbol{q}_{1}(\varepsilon)+a_{2} \boldsymbol{q}_{2}(\varepsilon)+a_{3} \boldsymbol{q}_{3}(\varepsilon)=a_{4} \boldsymbol{q}_{1}^{0}(\varepsilon)+a_{5} \boldsymbol{q}_{2}^{0}(\varepsilon)+a_{6} \boldsymbol{q}_{3}^{0}(\varepsilon), \quad \text { or } \quad \boldsymbol{L}_{\varepsilon} \boldsymbol{a}=0,
$$

where $\boldsymbol{a}=\left\{a_{1}, \ldots, a_{6}\right\}^{\mathrm{T}}$. The homogeneous system of six linear algebraic equations (3.2) has a non-trivial solution only if its determinant is equal to zero:

$$
W(k)=\operatorname{Det}\left\{\boldsymbol{L}_{\varepsilon}\right\}=0 .
$$

For a given set of governing parameters $R e_{l}, \eta, \zeta, W e, m, \omega$, dispersion relation (3.3) determines a set of eigenvalues $k_{i}$ as mentioned in $\S 2.3$. The solution of (3.3) can be carried out using the standard Newton-Raphson iterative algorithm. An initial guess for the eigenvalues $k_{0}$ is required in order to start calculations. Since the system of eigenvalues is not a priori known, we search the eigenvalues by varying the values of $k_{0}$ with a sufficiently small step over a wide domain in the complex $k$-plane. The verification of the numerical algorithm is presented in $\S 3.2$.

The eigenfunctions are expected to vary rapidly over the jet core $(r<1)$ and in the vicinity of the interface $(r \sim 1)$, while at the outer boundary of the flow $(r \gg 1)$ they tend to zero. In order to maintain reasonable accuracy of calculations, using mesh with a reasonably small number of nodes, we have implemented the variable mapping. The mesh is refined in the vicinity of the axis of the jet. The following mapping function $r(s)$ is used:

$$
r=e^{s}-r_{0}, \quad s=\ln \left(r+r_{0}\right), \quad \varepsilon \leqslant r \leqslant R .
$$

The value of parameter $r_{0}>0$ is determined based on the following conditions:

(i) one of the mesh nodes should coincide with the interface $r=1$;

(ii) there is a specified number of mesh nodes in the liquid jet core $N_{1}(r<1)$ and in the gas $N_{2}$. 


\begin{tabular}{ccc}
\multicolumn{2}{c}{ Current calculations } & Morris (1976) \\
$R=10$ & $R=50$ & \\
$0.2325+\mathrm{i} 0.0666$ & $0.2321+\mathrm{i} 0.0665$ & $0.2322+\mathrm{i} 0.0666$ \\
$0.3838+\mathrm{i} 0.3905$ & $0.3839+\mathrm{i} 0.3904$ & $0.3840+\mathrm{i} 0.3904$ \\
$0.4840+\mathrm{i} 0.8973$ & $0.4839+\mathrm{i} 0.8973$ & $0.4842+\mathrm{i} 0.8976$ \\
$0.5634+\mathrm{i} 1.5852$ & $0.5634+\mathrm{i} 1.5852$ & $0.5628+\mathrm{i} 1.5850$
\end{tabular}

TABLE 1. Comparison of calculated eigenvalues $k=k_{r}+\mathrm{i} k_{i}$ for $\varepsilon=10^{-3}, N_{1}=200$, $N_{2}=800$ with those reported by Morris (1976), $R e=80, m=0, \omega=0.2, N_{1}$ and $N_{2}$ are the numbers of mesh nodes in the jet core $(r<1)$ and surrounding gas $(1<r<R)$, respectively.

For a given set of parameters $\varepsilon, R, N_{1}$ and $N_{2}$, the value of $r_{0}$ is calculated using the Newton method.

The next stage involves the calculation of optimal disturbances based on the system of eigenfunctions. The integral over the computational domain involved in the expression for energy functional (2.36) is calculated numerically, and the energy matrix $\boldsymbol{E}(z)$ is obtained for a given downstream location $z$. The optimization problem for energy functional (2.41) is solved using a standard Lagrange-multiplier technique. The corresponding Lagrange function $P$ and Euler-Lagrange equations are presented as

$$
P=\boldsymbol{\gamma}^{*} \boldsymbol{E}(z) \boldsymbol{\gamma}+\lambda\left(\boldsymbol{\gamma}^{*} \boldsymbol{E}(0) \boldsymbol{\gamma}-1\right), \quad(\boldsymbol{E}(z)+\lambda \boldsymbol{E}(0)) \boldsymbol{\gamma}=0 .
$$

The second equation in system (3.5) is a generalized eigenvalue problem for positively defined Hermitian energy matrices evaluated at a starting location $z=0$ and at current position $z$. Eigenvector $\gamma$, which corresponds to the highest eigenvalue of (3.5), determines the optimal disturbance. Both the shape of the optimal disturbance and its energy depend on the location $z$ for a given set of governing parameters. Note that the jets under consideration are unstable for a wide range of governing parameters, and the first mode grows in the downstream direction. The energy of any combination of normal modes which involves the first mode would grow as well. Therefore, we studied the maximum optimal-to-single mode energy ratio if the flow is unstable at the specified set of governing parameters, and the actual optimal energy where this was not the case. Problem (3.5) is solved numerically using the $Q R$-algorithm (Wilkinson 1988).

\subsection{Verification of the numerical algorithm}

The numerical algorithm is tested for calculation of eigenvalues for the submerged jet with the 'smooth' velocity profile $U_{2}$. The comparison of calculated eigenvalues with those reported by Morris (1976) is presented in table 1. We note that only meridional modes are presented there.

Test calculations of normal modes have also been carried out for liquid jets in air, and it has been found that the convergence radius for the Newton iteration method, used to solve dispersion relation (3.3), is highly sensitive to the location of the inner boundary $r=\varepsilon$. When calculating the eigenvalues, we have considered the inner boundary to be sufficiently far from the axis $(\varepsilon \sim 0.1)$. Then we have performed iterations to obtain the eigenvalues for smaller $\varepsilon$. We have found that the desired accuracy of calculations is achieved for $R=50, \varepsilon=10^{-3}, N_{1}=200$ and $N_{2}=800$. 
Note that in stability calculations of unbounded flows the infinite flow domain is approximated by a finite flow domain, and therefore the continuous spectrum is represented by discrete modes (Butler \& Farrel 1992; Schmid \& Henningson 2001). Both discrete and continuous parts of the spectrum are obtained and used in calculations of optimal disturbances.

One of the main disadvantages of the orthonormalization method is that it requires the first guess of the complex wavenumber to be close enough to the eigenvalue, otherwise the algorithm will not converge (or converge to another neighbouring eigenvalue). The non-modal analysis involves the calculation of $N$ most unstable modes, and thorough calculations must be carried out in order not to overlook any normal modes, which would result in a reduction of the transient growth. The procedure for finding eigenvalues was cross-checked and customized by the results of spectra calculations using the $Q R$-algorithm (Gary \& Helgason 1970) implemented for a submerged jet. Another difficulty is in maintaining a reasonable accuracy of the calculations of higher (stable) modes (Schmid \& Henningson 2001). Test calculations showed that the absolute error of calculations of the 191st mode of the liquid jet in air with the velocity profile $U_{3}$ at $R e=10^{3}, \zeta=0.1, \eta=10^{-3}, W e=10^{3}$ with 1000 nodes $(k=0.1268+\mathrm{i} 11.922)$ is below $10^{-3}$, which we assumed to be sufficiently small.

A sensitivity study of the solution to the optimization problem (2.41) has been carried out. A typical dependence of the optimal-to-single mode energy ratio on the number of normal modes $N$ (see (2.28)) for a submerged jet is presented in figure 2(a) (the modes are ordered by the growth rate). It has been found that in the single-fluid case it is sufficient to consider $\sim 30$ modes in order to maintain reasonable accuracy of calculations.

As mentioned earlier, several authors have shown that the convergence of transient growth calculations for multiphase flows requires the interfacial energy term to be included into the norm. Test calculations of optimal disturbances with different values of parameter $M$ (see (2.36)) have been carried out. The results have shown that the inclusion of the interface energy term does not affect the convergence of transient growth calculations for liquid jets in air. All calculations performed with $M=0,0.01,0.1,1$ require around $N=200$ modes to converge (figure $2 b$ ). Note that the maximum difference between the values of optimal energy obtained at different $M$ is around $50 \%$. We are focused on analysing the physical phenomenon of transient growth in jets and, therefore, the choice of $M$ depends only on the convergence of our algorithm. In what follows we present the results of transient growth calculations at $M=0$. Additional calculations of transient growth have been carried out in the absence of the surface tension (interfacial terms in the boundary conditions at the jet interface and in the expression for energy were set equal to zero). The results are presented in figure $2(b)$. As follows from this figure, there is no convergence in the absence of the surface tension and interfacial energy terms. The optimal energy increases with an increase in the number of modes up to 200 . This qualitatively agrees with the results presented by South \& Hooper (1999).

\section{Results and discussion}

\subsection{Modal stability analysis}

The modal stability of submerged jets with 'smooth' velocity profile $U_{2}$ was analysed in a number of papers, including Batchelor \& Gill (1962) and Morris (1976). It was 


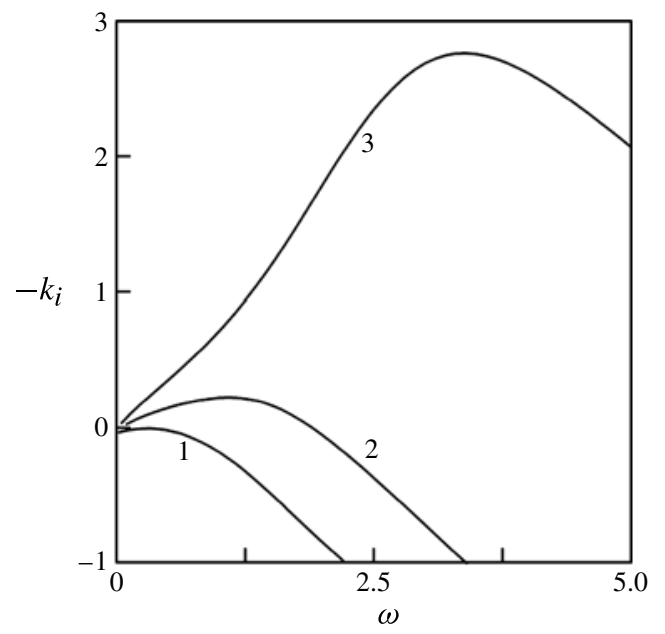

FIgURE 3. The growth rate of the first mode $-k_{i}$ against the frequency $\omega$ for the 'top-hat' velocity profile $U_{1}(2.13), m=0$. Curves $1-3$ correspond to $R e=20,50$ and 1000 .

found that jets with the velocity profile $U_{2}$ are stable with respect to axisymmetric disturbances $m=0$, and unstable at $m>0$ for $R e>38$.

In contrast to the 'smooth' jet, the 'top-hat' jet with the velocity profile $U_{1}$ is found to be unstable at any value of the azimuthal number. The critical Reynolds number value $R e_{c}$ is found to be $\sim 20$ (figure 3 ). This agrees with the results of calculations carried out by Morris (1976) for a qualitatively similar jet velocity profile. The submerged jets are unstable due to a shear-induced instability mechanism (Drazin \& Reid 1983).

It is found that the liquid jets in air described by velocity profiles $U_{3,4}$ are unstable at any Reynolds number due to the viscous analogue of the Kelvin-Helmholtz instability (Drazin \& Reid 1983). Typical plots for spatial growth rate $-k_{i}$ versus frequency $\omega$ for the least stable mode are presented in figure 4 . Note that there is a range in frequencies, over which non-axisymmetric disturbances $(m>0)$ grow faster than the axisymmetric disturbances $(m=0)$. Therefore, it is insufficient to consider only axisymmetric disturbances in order to describe the stability of liquid jets in air (Yang 1991). The maximum growth rate is gained by axisymmetric disturbances for both main-flow jet velocity profiles $U_{3,4}$.

\subsection{Non-modal stability analysis and optimal disturbances}

The maximization problem for energy functional (2.41), where the downstream location $z=z_{0}$ is a parameter, leads to the determination of the disturbance with maximum kinetic energy at a given position $z_{0}$ among all combinations (2.28). It is interesting to study optimal disturbances with the highest maximum energy gain among all values of the parameter $z_{0}$. Unless otherwise stated, in what follows only the results of calculations of global optimal disturbances, optimized over locations $z_{0}$, are presented. Note that, for the case when the jet is unstable, it is more informative to study the optimal-to-single mode energy ratio rather than the energies separately (they both grow in space).

The parametric study of global optimal disturbances to jets with profiles $U_{1-4}$ has been carried out. It is found that, for all velocity profiles under consideration, 

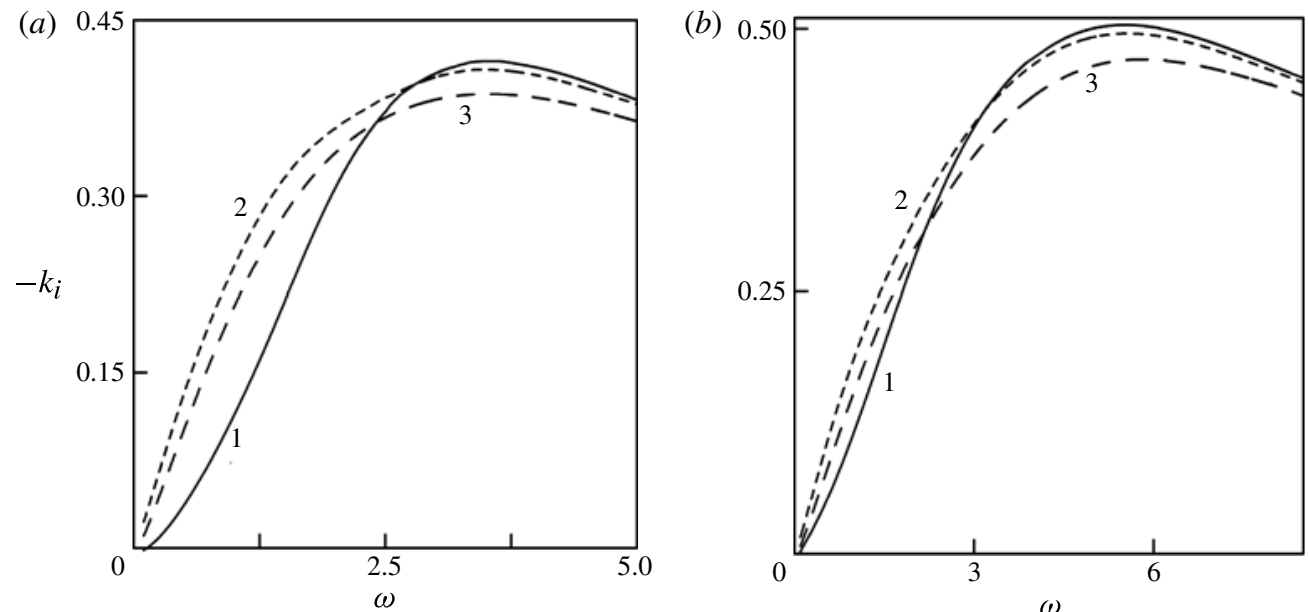

FIGURE 4. Growth rate of the first mode $-k_{i}$ against the frequency $\omega$ for jet velocity profiles $U_{3}(a)$ and $U_{4}(b)$. Curves $1-3$ correspond to $m=0,1$ and 2, respectively. Here $R e=1000$, $\eta=10^{-3}, \zeta=0.1$ and $W e=10^{3}$.
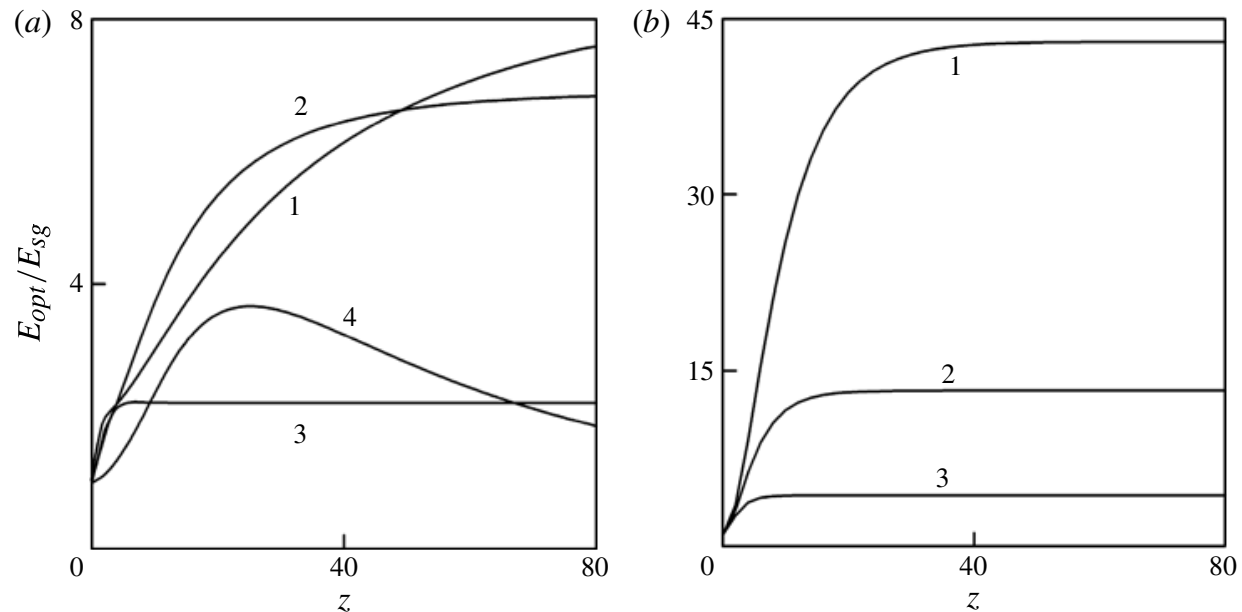

FIGURE 5. The ratio of optimal-to-single mode kinetic energy for a 'top-hat' jet velocity profile $U_{1}(2.13)$ versus the position downstream $z$ at $R e=1000$ for $m=0(a)$ and $m=1(b)$. Curves $1-4$ refer to $\omega=0.1,0.2,0.5$ and 0.0 , respectively. Growth rates $-k_{i}$ of the first mode are $-0.0003,0.0066,0.0288$ and 0.1921 for $1-4(a)$ and $0.0827,0.1582$ and 0.3451 for $1-3$ $(b)$.

the non-modal growth is significant only for non-axisymmetric disturbances $m>0$ (figures 5-7). Note that in figures 5-7 we presented the dependence of the optimalto-single mode energy ratio on $z$ with the largest maximum among all locations, where it is optimized. In other words, we presented the solution to the problem (2.41) optimized over locations $z$. The algebraic instability of submerged jets is stronger for the 'smooth' velocity profile $U_{2}$ in a wide range of frequencies $(\omega>0.1)$ and vice versa at $\omega=0$ (figures 5 and 6 ). Hence, at finite frequencies the non-modal growth 

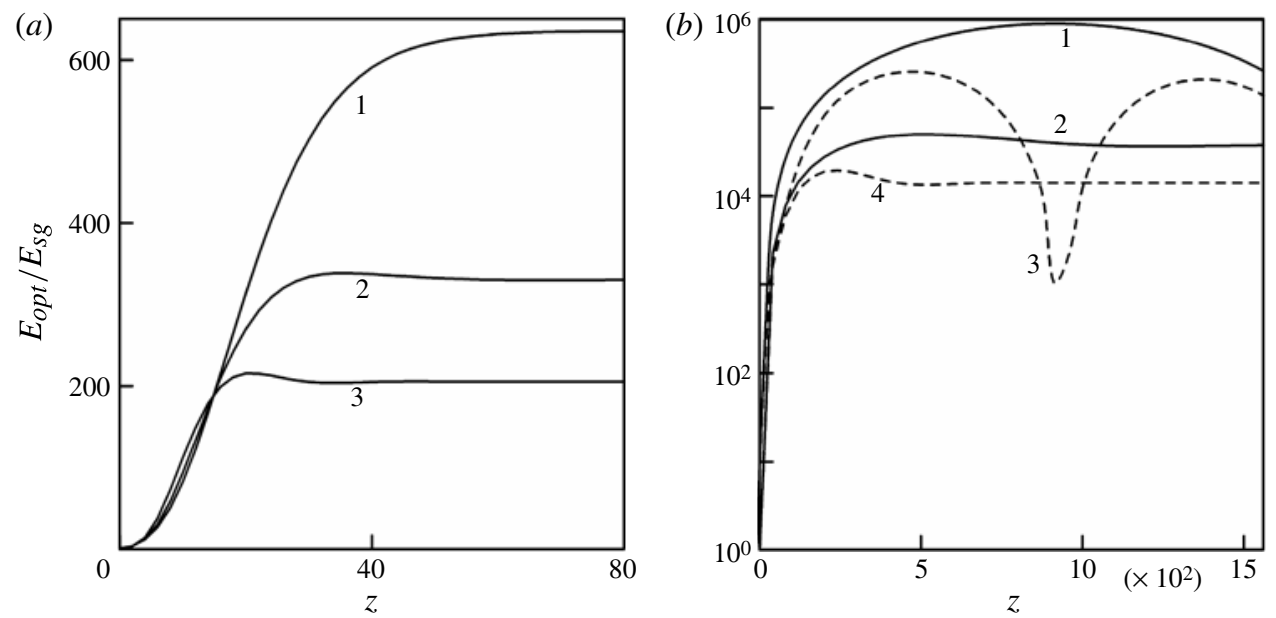

FIGURE 6. The ratio of optimal-to-single mode energy versus $z$ for a 'smooth' velocity profile $U_{2}(a)$ at $\omega=0.1,0.2$ and $0.5(1-3)$ and for profiles $U_{1}$ and $U_{2}(b)$ at $\omega=0(1,3)$ and $\omega=10^{-3}(2,4)$, respectively. Here $R e=1000, m=1$. Growth rates $-k_{i}$ of the first mode are $0.03,0.0471$ and 0.05 for $1-3(a)$ and $0.0,0.0,0.0002$ and 0.0004 for $1-4(b)$.
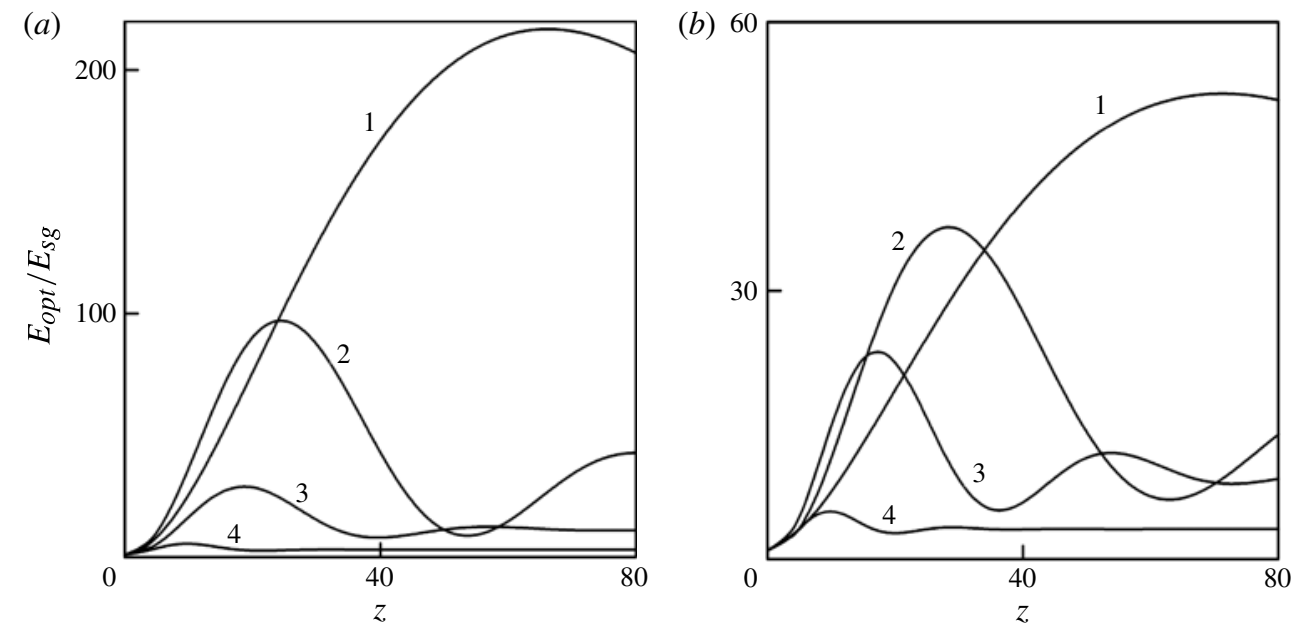

FIGURE 7. The optimal-to-single mode energy ratio against $z$ for jet velocity profiles $U_{3}$ (a) and $U_{4}(b)$. Curves 1-4 refer to $\omega=0,0.1,0.2$ and 0.5, respectively. Here $R e=1000$, $\eta=10^{-3}, \zeta=0.1, W e=10^{3}$ and $m=1$. Growth rates $-k_{i}$ of the first mode are 0.0, 0.0225, 0.0503 and 0.1321 for $1-4(a)$ and $0.0,0.016,0.0887$ and 0.0972 for $1-4(b)$.

of disturbances is more pronounced in the far-downstream region of the submerged jet. For the liquid jet in air, the optimal-to-single mode energy ratio oscillates in the downstream direction and levels off at large values of $z$, as the contribution of the least stable mode to the linear combination (2.28) dominates at $z \gg 1$ (figure 7). Note that although the modal instability of liquid jets in air and submerged jets are initiated by different mechanisms, the transient growth of disturbances to both jet types is strong. 
The growth rates of the first mode $-k_{i}$ are given in the captions to figures $5-7$. These rates allow us to estimate the actual growth of the optimal energy with $z$.

For the jet velocity profiles under consideration, the non-modal growth of nonaxisymmetric disturbances decreases with an increase in the frequency $\omega$ : the highest energy gain of optimal disturbances is gained at low frequencies $\omega \ll 1$ (figures 5-7). The non-modal instability mechanism weakens with an increase in $\omega$, which could be predicted a priori by analysing the dependence of normal modes on frequency $\omega$. Although the growth rate of the first mode increases over a wide range of frequencies (figures 3 and 4), all higher modes decay with an increase in $\omega$ as $k_{i} \sim \omega$. Therefore, energy of disturbance in the form of linear combination of modes (2.28) decreases at a given location $z$. Note that in order to study global optimal disturbances (with maximum energy gain at a given set of parameters $R e, \eta, \zeta$ and $W e$ ), optimization over frequency should be carried out. One of the main features of the jet spectra is that the wavenumbers of normal modes to both jet types at high Reynolds numbers are of the order of frequency $k_{r} \sim \omega$. A decrease in frequency below a certain threshold value results in an increase of wavelengths of disturbances above the jet relaxation length, and the plane-parallel approach used in the current study becomes inapplicable. In order to verify the non-modal analysis, the calculations at lower frequencies are also carried out as shown in figures $6(b)$ and 7. It is obtained that in submerged jets with profiles $U_{1,2}$ the maximum optimal-to-single mode energy ratio of steady disturbances $(\omega=0)$ is $E_{\text {opt }} / E_{s g} \sim 10^{6}$ and is gained far downstream from the nozzle $\left(z \sim 10^{3}\right)$. These results are in agreement with the calculations of spatial transient growth in boundary layers and pipes (Reshotko \& Tumin 2001; Tumin \& Reshotko 2001), where steady disturbances were shown to gain maximum non-modal amplification. Below we present the results of transient growth calculations for the sufficiently small but still finite frequency $\omega=0.1$, assuming that it is above the threshold value below which the plane-parallel approach is invalid.

The highest optimal-to-single mode energy ratio for both jet types at $R e=1000$, $\omega=0.1$ is of the order of $10^{2}$ (it is larger for submerged jets than for liquid jets). It is achieved at $z \sim 20$ for a wide range of parameters. As discussed in $\S 2.2$, at this distance the velocity profile varies insignificantly for $R e \gg 1$. Hence, we can assume that the instability parameters obtained are qualitatively similar to those for realistic jets.

The velocity distribution of the global optimal disturbance and that of the least stable mode with the same (unity) initial energy at $z=0$ have been studied. The velocity components of optimal disturbances and the least-stable mode for a liquid jet in air and submerged jets are shown in figures 8-11. Note that these velocity components are projections of the velocity vector to the basis vectors of the cylindrical coordinate system introduced earlier, but we use Cartesian coordinates $x=r \cos \theta, y=r \sin \theta$ to show their cross-section distribution. The cross-section velocity distribution of the least stable mode for the liquid jet in air is shown in figure 8 for the same parameters as in figure 7, curve 2. For a set of parameters under consideration, the flow is unstable, and the energy of all velocity components of the least stable mode increases exponentially with an increase in $z$. The shape of the initial distribution remains almost the same at all cross-sections downstream, therefore we present the cross-section distribution of the least-stable mode only at location $z=20$, where the corresponding optimal perturbation gains maximum energy. The development of the optimal disturbances is different from that of the least stable mode, as shown in figure 9 . The initial shapes of the streamwise velocity component $u$ are similar for the first mode and the global optimal disturbance (figures $8 a$ and $9 a$ ). 

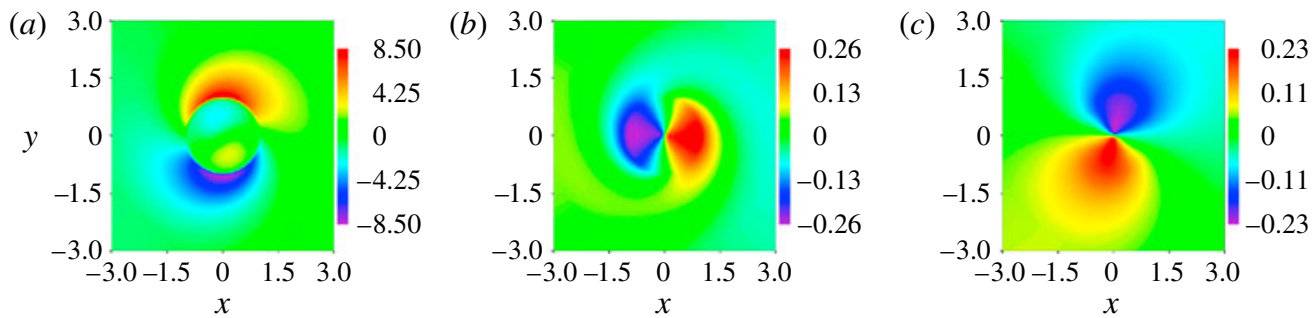

FIGURE 8. Cross-sectional distributions of velocity components $u$ (along $z, a$ ), $v$ (along $r, b$ ), and $w$ (along $\theta, c$ ) for the least stable mode of the liquid jet in air with velocity profile $U_{3}$, $z=20$. Parameters used for calculations are the same as those for figure 7, curve 2. Cartesian coordinates $x=r \cos (\theta), y=r \sin (\theta)$ are used.
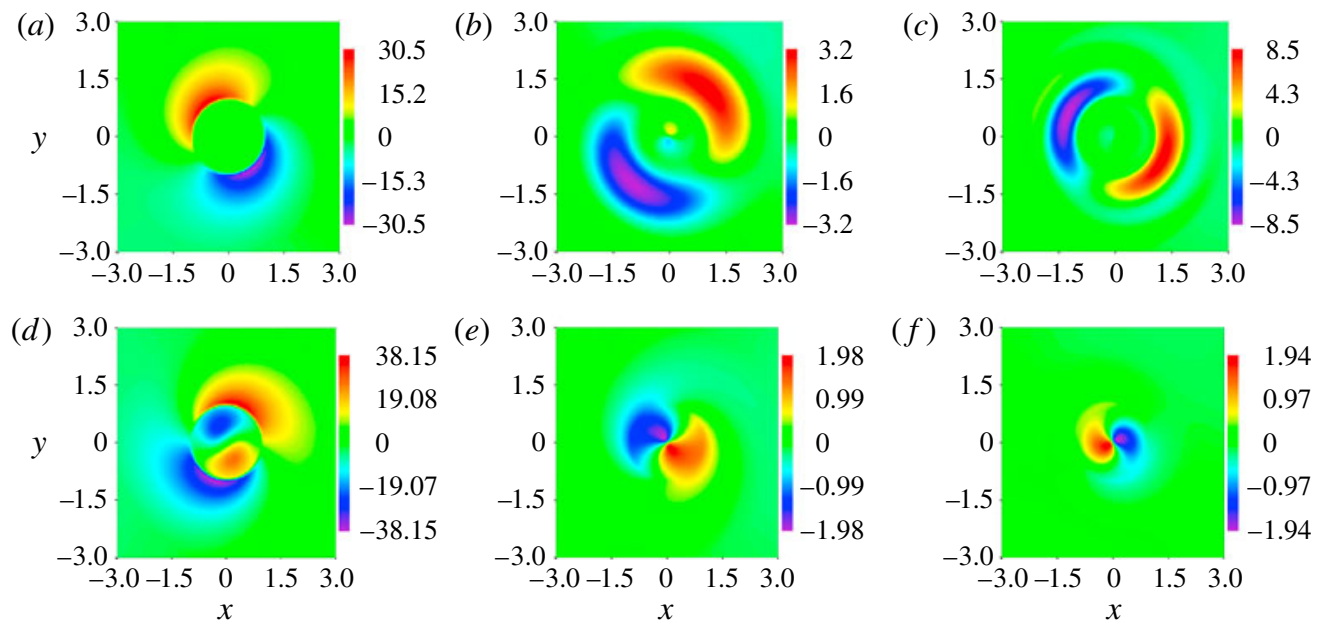

FIGURE 9. Cross-section distributions of velocity components $u$ (along $z ; a, d$ ), $v$ (along $r$; $b, e$ ), and $w$ (along $\theta ; c, f$ ) for the optimal disturbance to a liquid jet in air with velocity profile $U_{3}$ at $z=0$ (upper plots) and $z=20$ (lower plots). Parameters used for calculations are the same as those for figure 7, curve 2. Cartesian coordinates $x=r \cos (\theta), y=r \sin (\theta)$ are used.

The distributions of $v$ - and $w$-velocity components are different for these two cases: in the case of the least stable mode, the energy of $v$ - and $w$-velocity components are concentrated in the jet fluid (figure $8 b, c$ ), while in the case of the optimal disturbance these velocity components are initially concentrated in the ambient gas (figure $9 b, c$ ). With an increase in $z$, the energy of $v$ - and $w$-velocity components is transformed into the energy of the $u$-component (compare the upper and lower plots in figure 9). Note that in the optimal perturbation, it is only the $u$-velocity component of the jet liquid which grows significantly due to the energy transfer from other velocity components, as the $u$-distribution of the surrounding gas does not change significantly with an increase in $z$ (figure $9 a, d$ ). The same scenario of the optimal disturbance development is found in the case of a submerged jet (figures 10 and 11). The energy of all velocity components of the optimal perturbation increases with an increase in $z$. The streamwise velocity gains larger growth than the other components: the maximum of $u$ 

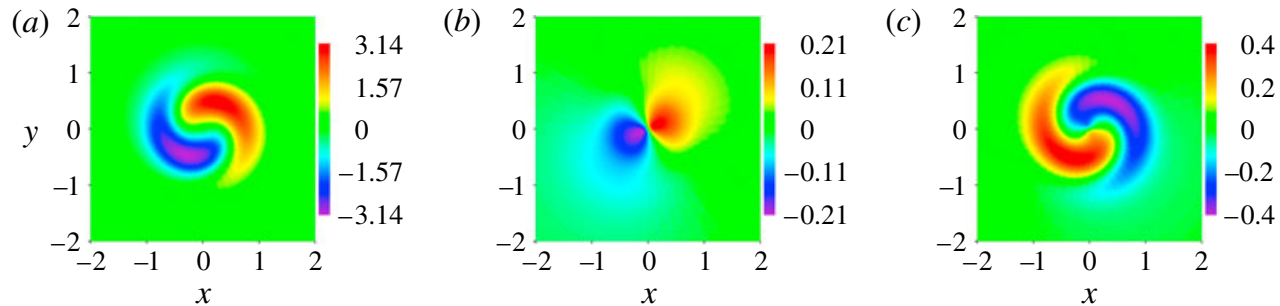

FIGURE 10. Cross-section distributions of velocity components $u$ (along $z ; a$ ), $v$ (along $r ; b$ ) and $w$ (along $\theta ; c$ ) for the least stable mode of the submerged jet with the velocity profile $U_{2}$, $z=20, \operatorname{Re}=1000, \omega=0.1$ and $m=1$. Cartesian coordinates $x=r \cos \theta, y=r \sin \theta$ are used.

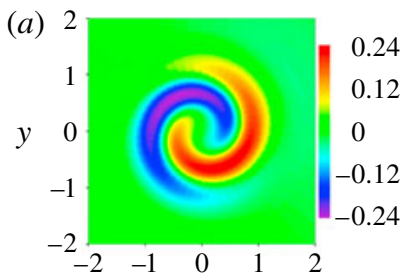

(b)
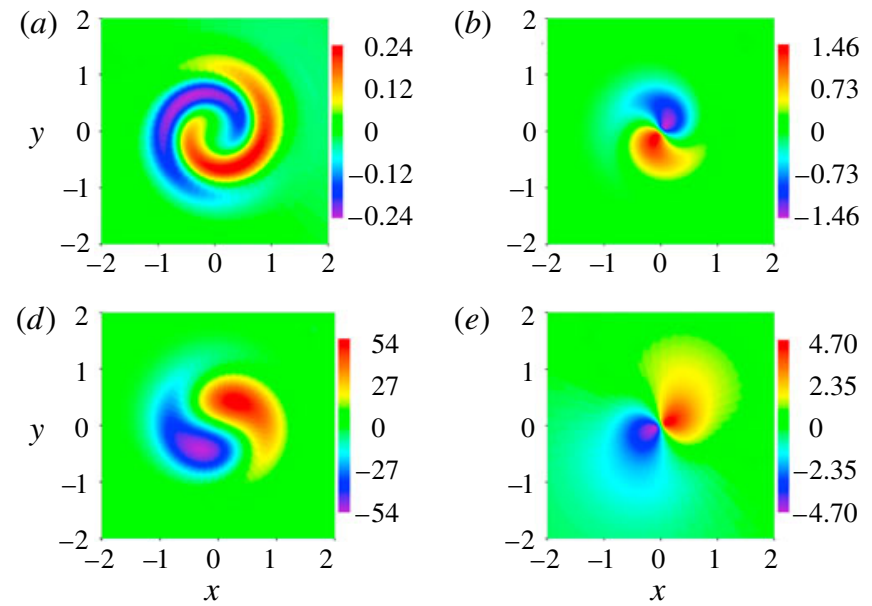

(e)

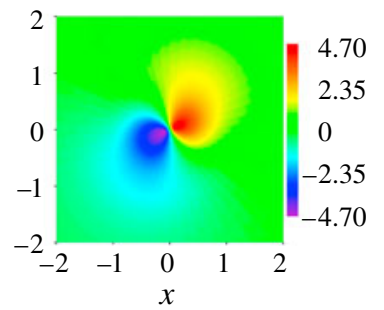

(c)

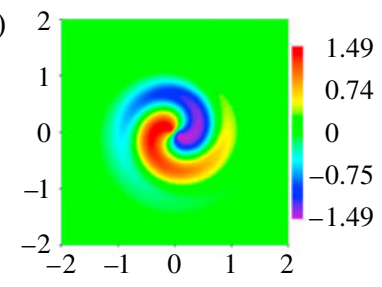

$(f)$

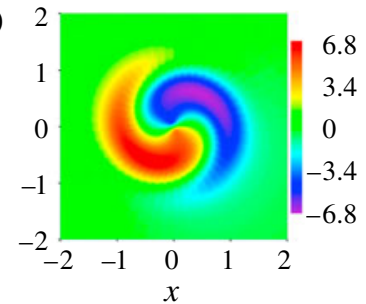

FIGURE 11. Cross-section distributions of velocity components $u$ (along $z ; a, d$ ), $v$ (along $r ; b, e)$ and $w$ (along $\theta ; c, f$ ) for the optimal disturbance to a submerged jet with velocity profile $U_{2}$ at $z=0(a-c)$ and $z=20(d-f)$, with $R e=1000, \omega=0.1$ and $m=1$. Cartesian coordinates $x=r \cos (\theta), y=r \sin (\theta)$ are used.

is increased by two orders of magnitude, while maximums of $v$ and $w$ are increased only four-fold (figure 11).

The amplification of the streamwise velocity component of the jet is due to the 'lift-up' (or 'vortex-tilting') instability mechanism which was found in various shear flows (Ellingsen 1975; Landahl 1980; Butler \& Farrel 1992; Reshotko \& Tumin 2001; Tumin \& Reshotko 2001; Yecko \& Zaleski 2005; Schmid 2007). This mechanism is easily demonstrated for a plane inviscid flow with velocity profile $U(y)$. If we consider the streamwise-independent small disturbance to a basic shear flow, then according to the linearized Euler equations, the normal velocity $v$ is independent of time and the streamwise velocity component $u$ grows linearly with time (Ellingsen 1975). Alternatively, from the linearized inviscid vorticity equation, it is obtained that the $y$-component of the vorticity disturbance grows with time proportionally to the $z$-component of the basic flow vorticity meaning that the main vorticity is tilted in the $y$-direction (Butler \& Farrel 1992). 


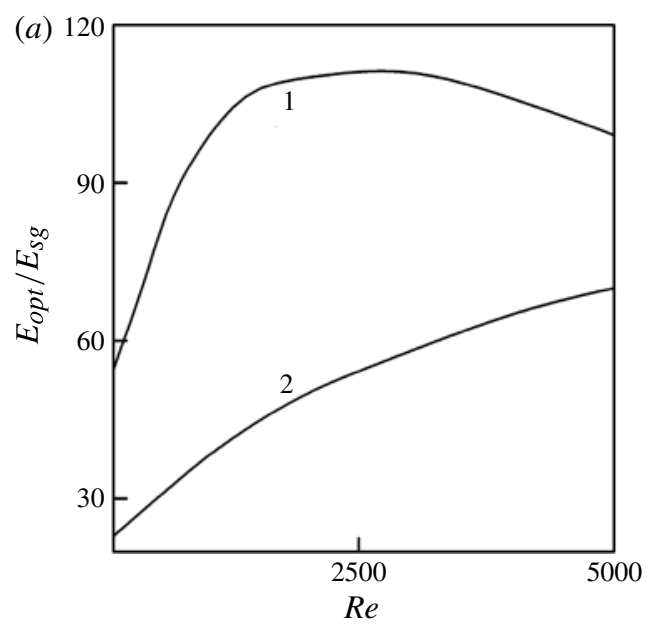

(b)

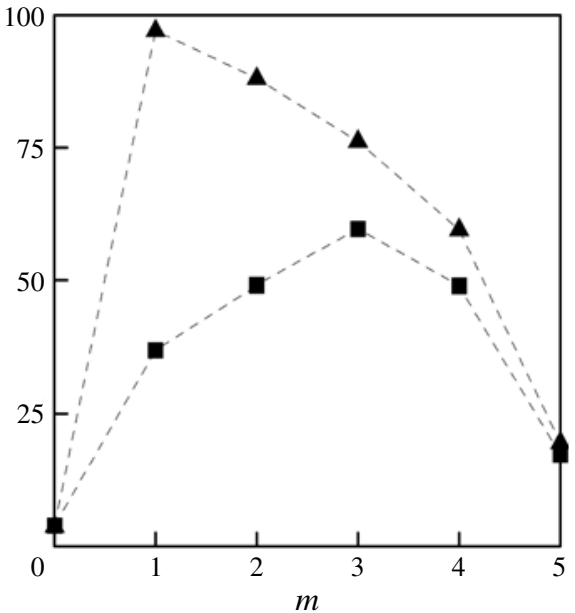

FIGURE 12. Optimal-to-single mode energy ratio against the Reynolds number at $m=1(a)$ and azimuthal mode number $m$ at $R e=1000(b)$ for liquid jets in air with velocity profiles $U_{3}$ (curve 1 and triangles) and $U_{4}$ (curve 2 and squares). Here $\eta=10^{-3}, \zeta=0.1, W e=10^{3}$ and $\omega=0.1$.

Dependence of optimal perturbations on the Reynolds number $R e$ has been studied. In a number of studies devoted to the non-modal stability of plane-parallel flows (Butler \& Farrel 1992; Reshotko \& Tumin 2001; Tumin \& Reshotko 2001; Yecko \& Zaleski 2005), it was found that the energy of global optimal disturbances increases by a factor of $R e^{2}$ with an increase in the Reynolds number. The maximum (over $z$ ) energy of optimal disturbances to jets also increases with an increase in $R e$ at $\omega=0.1$ given that all other parameters are fixed, although the dependence is not quadratic (figure 12a). For a jet with profile $U_{3}$ the optimal-to-single mode energy reaches a maximum over $\operatorname{Re}$ and $z$ at $R e \sim 2500$ and decreases at higher Reynolds numbers. It is found that the optimal-to-single mode energy ratio of a jet with profile $U_{4}$ increases up to $R e=5000$. Note that we study the optimal-to-single mode energy ratio rather than the optimal energy itself, since jets are unstable in a wide range of governing parameters. The growth rate of the first mode increases with an increase in the Reynolds number, and it leads to the weakening of transient growth compared with the energy growth of the first mode. Since the quadratic increase in global optimal energy is a unique feature of the transient growth of disturbances to all shear flows, we carried out calculations of global optimal disturbances to a jet with profile $U_{3}$ at $m=1, \zeta=0.1, \eta=10^{-3}, W e=10^{3}, R e=1000$ and 2000 (global optimal disturbances are steady $\omega=0$ and the growth rate of the first mode is zero $\left.k_{i}=0\right)$. The global optimal energy values turned out to be 217 and 869, respectively (a two-fold increase in $R e$ results in a four-fold increase in energy), which confirms the quadratic Reynolds number scaling of optimal energy in jets.

Calculations of non-axisymmetric optimal perturbations with different azimuthal mode numbers $(m>1)$ have been carried out. It is found that for a given set of parameters the most pronounced non-modal growth is gained by disturbances with $m=1-4$, an increase in $m$ leads to a decrease in the maximum energy of optimal disturbances (figure 12b). Note that for $m=4,5$ first modes for liquid jets with both velocity profiles $U_{3,4}$ decay. Therefore, for these values of $m$, the actual maximum 
values of the optimal energy $E_{\text {opt }}(z)$ normalised by its values at $z=0$ are plotted. Note that the direct comparison of the transient growth at different $m$ shown in figure $12(b)$ is impossible without considering the dependence of the growth rate of the first mode on $m$. Note that the optimal-to-single mode energy ratio calculated for a jet with velocity profile $U_{4}$ at $m=1\left(E_{\text {opt }} / E_{s g}=37\right)$ is lower than that at $m=2\left(E_{\text {opt }} / E_{s g}=49\right)$, which in turn is lower than the energy ratio calculated at $m=3\left(E_{\text {opt }} / E_{s g}=60\right)$. The corresponding growth rates of the first mode at $m=1,2$ and 3 are $k_{i}=-0.016$, -0.0076 and -0.0007 , respectively. The highest optimal energy is gained at $z \sim 20$ for all three cases, and comparing the actual values of optimal energies we find that the highest energy is gained by the optimal disturbance with $m=1$, while at $m=3$ the optimal energy value is lowest. The difference between the actual values of optimal energy is around 5\%, and we expect that all disturbances with $m=1-3$ can contribute to the jet break-up depending on the configuration of initial disturbances (at $z=0$ ).

The significant difference between this and other theoretical studies of non-modal stability of plane-parallel flows published in the open literature is that liquid jets in air are unstable at any Reynolds number. Typical growth rates of small disturbances in two-phase jets are quite high, which makes it difficult to provide a direct comparison of the current results with existing experimental data. In the plane-parallel flows, such as plane-channel flow, a subcritical transient growth of disturbances can be initiated by a certain initial (optimal) perturbation. In the case of a liquid jet in air and submerged jet at high Reynolds number, any disturbances would grow, and it will not be easy to distinguish between modal and non-modal instabilities. However, the results of the current study are qualitatively confirmed by several experimental studies on jet break-up and theoretical studies on non-modal instability of the two-phase mixing layer. As discussed earlier, the strongest non-modal growth is obtained at $m=1-5$, and optimal disturbances are non-axisymmetric. The non-axisymmetry of jet break-up at high Reynolds numbers ( $R e>100$ ) was pointed out by Hoyt \& Taylor (1977), Lin \& Reitz (1998) and Lasheras \& Hopfinger (2000). Marmottant \& Villermaux (2004) suggested the following estimate for the wavelength of transverse disturbances to a jet

$$
\lambda_{\perp} \sim \delta W e_{\delta}^{-1 / 3}\left(\rho_{1} / \rho_{2}\right)^{1 / 3},
$$

where $\delta$ is the vorticity layer thickness in air, $W e_{\delta}$ is calculated using gas velocity at the interface and $\delta$, and $\rho_{1}, \rho_{2}$ are densities of the jet liquid and air. Results of calculations presented in figure $12 b$ refer to $\delta \sim 0.2, W e_{\delta} \sim 50$ and $\rho_{1} / \rho_{2}=10^{3}$. Formula (4.1) gives $\lambda_{\perp} \sim 0.5$, where $\lambda_{\perp}$ is non-dimensionalised by the jet radius $L$, which corresponds to $m \sim 12$. This qualitatively agrees with the predicted azimuthal mode numbers with a significant transient growth $m=1-5$, shown in figure $12 b$. Note that the jet configuration studied by Marmottant \& Villermaux (2004) is different from that investigated in the current study: the focus of their analysis was on a jet with a high-speed coflowing axial gas stream, while we considered the instability of a jet released into a stagnant medium. Another source of discrepancy is that we used different velocity profiles. As has been shown both theoretically and experimentally, the number of ligaments does not depend on the liquid-to-gas shear layer width (Yecko \& Zaleski 2005). This is confirmed by our calculations: the dependence of transient growth on the azimuthal mode number $m$ is similar for both velocity profiles $U_{3}$ and $U_{4}$, which differ by the liquid shear layer width only. We found, however, that the non-modal growth is more pronounced for the jet with the velocity profile $U_{3}$, for which the liquid shear layer width is larger than that for the profile $U_{4}$. 


\section{Conclusions}

Transient growth of disturbances to round jets has been considered here for the first time. The systems of three-dimensional normal modes have been calculated for submerged jets and liquid jets in air with various velocity profiles. Disturbances which maximize the kinetic energy functional at a given position downstream are found. For all jet velocity profiles under consideration, the non-modal growth of disturbances is shown to be significant: the energy gain of optimal disturbances at a Reynolds number of 1000 is up to two orders of magnitude larger than that of the least stable single mode at the location downstream which is equivalent to about 20 diameters of the jet. The non-modal growth is significant only in the case of non-axisymmetric disturbances. The strongest algebraic instability is gained by steady disturbances; the transient growth mechanism weakens relative to the exponential growth of the first mode with an increase in frequency and decrease in Reynolds number. The streamwise velocity component of optimal disturbances to both jet types gains significantly larger growth compared with other velocity components due to the 'lift-up' mechanism.

In the case of a liquid jet in air and submerged jet at high Reynolds number, there is a certain range of frequencies which correspond to growing disturbances and it is not easy to distinguish between modal and non-modal instabilities. The results of the current study, however, are consistent with several experimental studies on jet break-up and theoretical studies of non-modal instabilities of a two-phase mixing layer. The strongest non-modal growth is obtained at $m=1-5$, and optimal disturbances are shown to be non-axisymmetric. In agreement with the previous studies it is shown that the dependence of transient growth on the azimuthal mode number $m$ is similar for both velocity profiles $U_{3}$ and $U_{4}$, which differ by the liquid shear layer width only. The non-modal growth is shown to be more pronounced for the jet with the velocity profile $U_{3}$, for which the liquid shear layer width is larger than that for the profile $U_{4}$.

\section{Acknowledgements}

The authors are grateful to Professor A. Osiptsov for useful discussions of the problem, to EPSRC (grants EP/F069855/1, EP/F069855/1 and EP/H001603/1), RFBR (grants 11-01-00483 and 12-08-31420) and the Russian Ministry of Education and Science (grant for young Russian PhD researchers MK-3582.2011.1) for the financial support of this project.

\section{Appendix A.}

The main flow velocity profiles $U_{3,4}$ for a liquid jet in air are approximated as

$$
\begin{aligned}
& U_{l}(r)=\left\{\begin{array}{ll}
1, & r \leqslant r_{l} \\
\alpha_{l} \exp \left\{-\left(r-r_{l}\right)^{2} / \delta_{l}^{2}\right\}+\beta_{l}, & r_{l}<r<1
\end{array},\right. \\
& U_{g}(r)=\alpha_{g} \exp \left\{\frac{-\left(r-r_{g}\right)^{2}}{\delta_{g}^{2}}\right\}, \quad r>1 .
\end{aligned}
$$

The values of parameters $\alpha_{l}, \beta_{l}, r_{l}$ and $\delta_{l}$, are presented in table 2 , and the values of $\alpha_{g}, r_{g}$ are determined based on the conditions at the interface (see (2.15)).

Velocity profiles depend on the gas-to-liquid viscosity ratio $\zeta$. We have taken $\delta_{g}=0.14$ for $\zeta=10^{-1}$ and $\delta_{g}=0.25$ for $\zeta=10^{-2}$ so that the shear layer of the surrounding gas in the former case is around $\sqrt{10}$ times wider than that in the latter 


$\begin{array}{ccccc} & \alpha_{l} & \beta_{l} & r_{l} & \delta_{l} \\ U_{3}(r) & 0.6 & 0.4 & 0 & 0.7 \\ U_{4}(r) & 0.4 & 0.6 & 0.5 & 0.4\end{array}$

TABLE 2. Parameters of velocity profiles for the liquid jet in air $U_{3,4}$ determined by expressions (A 1), (A 2) and plotted in figure 1.

case (we assume that the shear layer width is scaled as a square root of the Reynolds number, just as it is scaled for the boundary layer over the flat plate).

\section{Appendix B.}

Linearized equations (2.16)-(2.19), in terms of the amplitudes of disturbances in the form of travelling waves, are presented as (Batchelor \& Gill 1962)

$$
\left.\begin{array}{rl}
\mathrm{i} k u+v^{\prime}+\frac{v}{r}+\mathrm{i} m \frac{w}{r} & =0, \quad(\mathrm{i} k U-\omega) u+U^{\prime} v=-\mathrm{i} k p+\frac{1}{R e} \Delta u, \\
(\mathrm{i} k U-\omega) v & =-p^{\prime}+\frac{1}{R e}\left(\Delta v-\frac{2 \mathrm{i} m}{r^{2}} w\right), \\
(\mathrm{i} k U-\omega) w & =-\frac{i m}{r} p+\frac{1}{R e}\left(\Delta w+\frac{2 \mathrm{i} m}{r^{2}} v\right),
\end{array}\right\}
$$

where

$$
\Delta=\frac{\mathrm{d}^{2}}{\mathrm{~d} r^{2}}+\frac{1}{r} \frac{\mathrm{d}}{\mathrm{d} r}-\left(k^{2}+\frac{m^{2}+1}{r^{2}}\right) .
$$

Boundary conditions (2.20)-(2.24) can be presented as

$$
\left.\begin{array}{c}
\mathrm{i} h\left(k U_{\alpha}-\omega\right)=v_{\alpha}(\alpha=g, l), \quad u_{l}+h U_{l}^{\prime}=u_{g}+h U_{g}^{\prime}, \quad v_{l}=v_{g}, \quad w_{l}=w_{g}, \\
\mathrm{i} k v_{l}+u_{l}^{\prime}+h U_{l}^{\prime \prime}=\zeta\left(\mathrm{i} k v_{g}+u_{g}^{\prime}+h U_{g}^{\prime \prime}\right), \\
-p_{l}+\frac{2}{R e_{l}} v_{l}^{\prime}=-\eta p_{g}+\frac{2 \zeta}{R e_{l}} v_{g}^{\prime}+\frac{h}{W e}\left(1-k^{2}-m^{2}\right) \\
w_{l}^{\prime}+\mathrm{i} m v_{l}-w_{l}=\zeta\left(w_{g}^{\prime}+\mathrm{i} m v_{g}-w_{g}\right) .
\end{array}\right\}
$$

\section{REFERENCES}

Batchelor, G. K. 1967 An Introduction to Fluid Dynamics. Cambridge University Press.

BAtchelor, G. K. \& Gill, A. E. 1962 Analysis of the stability of axisymmetric jets. J. Fluid Mech. 14, 529-551.

Butler, K. M. \& FARrel, D. F. 1992 Three-dimensional optimal perturbations in viscous shear flow. Phys. Fluids A 4 (5), 1637-1650.

Crua, C., Kennaird, D. A., Sazhin, S. S., Heikal, M. R. \& Gold, M. R. 2004 Diesel autoignition at elevated in-cylinder pressures. Intl J. Engine Res. 5 (4), 365-374.

DE LuCA, L. 2001 Non-modal growth of disturbances in free-surface flows. In Proceedings of International Conference RDAMM-2001, 6(2).

Duda, J. L. \& VRentas, J. S. 1967 Fluid mechanics of laminar liquid jets. Chem. Engng Sci. 22, $855-869$.

Drazin, P. G. \& ReID, W. H. 1983 Hydrodynamic Stability, 2nd edn. Cambridge University Press.

Ellingsen, T. 1975 Stability of linear flow. Phys. Fluids 18, 487-488. 
Funada, T., Joseph, D. D. \& Yamashita, S. 2004 Stability of a liquid jet into incompressible gases and liquids. Intl J. Multiphase Flow 30, 1279-1310.

GARG, V. K. \& Rouleau, W. T. 1971 Linear spatial stability of pipe Poiseuille flow. J. Fluid Mech. 54, 113-127.

GARY, J. \& HELgason, R. 1970 A matrix method for ordinary differential eigenvalue problems. J. Comput. Phys. 5, 169-187.

GodUNOV, S. K. 1961 Numerical solution of boundary-value problems for the systems of linear ordinary differential equations. Usp. Mat. Nauk 16 (3), 171-174.

Hoyt, J. W. \& TaYlor, J. J. 1977 Waves on water jets. J. Fluid Mech. 83, 119-127.

KLingmanN, G. B. 1992 On transition due to three-dimensional disturbances in plane Poiseuille flow. J. Fluid Mech. 240, 167-195.

LANDAHL, M. L. 1980 A note on algebraic instability of inviscid parallel shear flows. J. Fluid Mech. 98, 243-251.

Landau, L. D. \& Lifshitz, E. M. 1959 Fluid Mechanics, 2nd edn. Pergamon.

Lasheras, J. C. \& Hopfinger, E. J. 2000 Liquid jet instability and atomization in a coaxial gas stream. Annu. Rev. Fluid Mech. 32, 275-308.

Levin, O., Chernoray, V. G., Löfdahl, L. \& Henningson, D. S. 2005 A study of the Blasius wall jet. J. Fluid Mech. 539, 313-347.

Lin, S. P. \& Reitz, R. D. 1998 Drop and spray formation from a liquid jet. Annu. Rev. Fluid Mech. 30, 85-105.

MAlik, S. V. \& Hooper, A. P. 2007 Three-dimensional disturbances in channel flows. Phys. Fluids 19, 052102.

Marmottant, P. \& Villermaux, E. 2004 On spray formation. J. Fluid Mech. 498, 73-111.

MAYER, W. O. H. 1994 Coaxial atomization of a round liquid jet in a high speed gas stream: A phenomenological study. Exp. Fluids 16, 401-410.

MORRIS, P. J. 1976 The spatial viscous instability of axisymmetric jets. J. Fluid Mech. 77, 511-529.

VAn Noorden, T. L., Boomkamp, P. A. M., KnaAp, M. C. \& Verheggen, T. M. M. 1998 Transient growth in parallel two-phase flow: analogies and differences with single-phase flow. Phys. Fluids 10 (8), 2099-2101.

RaYleigh, J. W. S. 1945 The Theory of Sound, vol. II. 2nd edn. Dover.

RENARDY, Y. 1987 The thin-layer effect and interfacial stability in a two-layer Couette flow with similar liquids. Phys. Fluids 30 (6), 1627-1637.

Reshotko, E. \& Tumin, A. 2001 Spatial theory of optimal disturbances in a circular pipe flow. Phys. Fluids 13 (4), 991-996.

REYNOLDS, O. 1883 An experimental investigation of the circumstances which determine whether the motion of water shall be direct or sinuous, and of the law of resistance in parallel channels. Phil. Trans. 174, 935-982.

Sazhin, S. S., Crua, C., Kennaird, D. A. \& Heikal, M. R. 2003 The initial stage of fuel spray penetration. Fuel 82 (8), 875-885.

Sazhin, S. S., Martynov, S. B., Kristyadi, T., Crua, C. \& Heikal, M. R. 2008 Diesel fuel spray penetration, heating, evapouration and ignition: modelling versus experimentation. Intl $J$. Engng Syst. Modelling Simul. 1 (1), 1-19.

Schmid, P. J. \& Henningson, D. S. 2001 Stability and Transition in Shear Flows. Springer.

SCHMid, P. J. 2007 Non-modal stability theory. Annu. Rev. Fluid Mech. 39, 129-162.

ShKadov, V. YA. \& Sisoev, G. M. 1996 Instability of a two-layer capillary jet. Intl J. Multiphase Flow 22 (2), 363-377.

Söderberg, L. D. \& Alfredsson, P. H. 1998 Experimental and theoretical investigations of plane liquid jets. Eur. J. Mech. B/Fluids 17 (5), 689-737.

South, M. J. \& Hooper, A. P. 1999 Linear growth in two-fluid plane Poiseuille flow. J. Fluid Mech. 381, 121-139.

TUMin, A. \& REShotKo, E. 2001 Spatial theory of optimal disturbances in boundary layers. Phys. Fluids 13 (7), 2097-2104. 
Turner, M. R., Healey, J. J., SAZhin, S. S. \& Piazzesi, R. $2012 a$ Wave packet analysis and break-up length calculations for an accelerating planar liquid jet. Fluid Dyn. Res. 44, 015503 26pp.

Turner, M. R., Sazhin, S. S., Healey, J. J., Crua, C. \& Martynov, S. B. $2012 b$ A breakup model for transient diesel fuel sprays. Fuel 97, 288-305.

Wilkinson, J. H. 1988 Algebraic eigenvalue problem. Clarendon Press.

YANG, H. Q. 1991 Asymmetric instability of a liquid jet. Phys. Fluids 4 (4), 681-689.

Yecko, P. A. \& ZALESKi, S. 2005 Transient growth in two-phase mixing layers. J. Fluid Mech. 528, 43-52. 